\title{
Expression of cardiac insulin signalling genes and proteins in rats fed a high-sucrose diet: effect of bilberry anthocyanin extract
}

\author{
Shamjeet Singh ${ }^{1}$, Thomas Netticadan ${ }^{2}$ and D. Dan Ramdath ${ }^{1,3^{*}}$
}

\begin{abstract}
Scope: Insulin resistance is associated with impaired cardiac function, but the underlying molecular abnormalities are largely unexplained. Bilberry anthocyanin (BAcn) may be protective, as it appears to potentiate insulin action.

Methods: Rats were randomly allocated to control, sucrose-fed (SF) or sucrose-fed + BAcn diets (SF-A) for 15 weeks. Cardiac insulin signalling genes and proteins were quantified using reverse transcription quantitative real-time polymerase chain reaction and western blots.

Results: Glucose tolerance was not different with treatment. SF showed lower $(p<0.05)$ ferric reducing antioxidant power, which increased with BAcn. SF resulted in significantly decreased $(p<0.05)$ expression of 10 genes: acetyl-coenzyme A carboxylase alpha; V-Akt murine thymoma viral oncogene homolog 1; Bcl2-like 1; cytosine-cytosine-adenosine-adenosine-thymidine/enhancer binding protein; FK506 binding protein 12-rapamycin associated; glycerol-3-phosphate dehydrogenase 1 (soluble); solute carrier family 2 (facilitated glucose transporter), member 1, 4; hexokinase 2; and thyroglobulin. SF-A prevented these changes. Compared to SF-A, SF up-regulated $(p<0.05)$ complement factor $\mathrm{D}$ and phosphoinositide-3-kinase, regulatory subunit1 ( $a)$; sterol regulatory element binding transcription factor 1 was down-regulated $(p<0.05)$. SF increased $(p<0.05)$ cardiac phospholamban and decreased phosphorylated troponin I, which were not attenuated by BAcn. Compared to control or SF, SF-A resulted in significantly lower $(p<0.05)$ 5'-AMP-activated protein kinase.
\end{abstract}

Conclusions: SF lowered antioxidant capacity and changed the expression of insulin signalling genes, which were modulated by BAcn.

Keywords: Anthocyanins, Heart, Insulin resistance, PCR array, Type 2 diabetes mellitus

\section{Introduction}

Increased consumption of diets high in refined carbohydrates has been linked to the growing prevalence of type 2 diabetes mellitus (T2DM) (Eckel 2005; Mellor et al. 2010). Current estimates suggest that approximately $6.6 \%$ of the world's adult population is diabetic with the majority having T2DM (IDF 2009). Of greater

\footnotetext{
* Correspondence: dan.ramdath@agr.gc.ca

${ }^{1}$ Department of Pre-Clinical Sciences, Faculty of Medical Sciences, The University of the West Indies, St. Augustine Campus, St. Augustine, Trinidad and Tobago, West Indies

${ }^{3}$ Guelph Food Research Centre, Agriculture and Agri-Food Canada, 93 Stone Road West, Guelph, ON N1G 5C9, Canada

Full list of author information is available at the end of the article
}

concern is that about $7.9 \%$ show impaired glucose tolerance (IGT) or prediabetes (IDF 2009). One of the main underlying cellular defects associated with the diabetic and prediabetic state is insulin resistance (IR) (Abdul-Ghani and DeFronzo 2009; Reaven 1988), which is the inability of cells to respond to the action of insulin and can exist several years prior to the onset of T2DM (Lebovitz 1999).

IR can induce debilitating effects on various organs such as the kidneys, brain, liver and especially the heart (Sharma and McNeill 2006). In fact, epidemiological and clinical studies suggest that a large percentage of diabetics succumb to cardiac complications (Asghar 
et al. 2009), and this is largely attributed to myocardial infarction resulting from atherosclerotic build-up (DeFronzo 2010). However, the existence of a distinct disease of the heart muscle known as diabetic cardiomyopathy (DCM) has also been demonstrated (Galderisi et al. 1991). Although much is unknown about the aetiology of DCM, it can play a significant role in heart failure and in the recovery of cardiac tissue after an ischemic insult (Boudina and Abel 2007; Maisch et al. 2011). It is therefore crucial to examine the underlying cellular defects that accompany the very early stages of the development of IR and its contribution to the aetiology of DCM.

In the normal heart, binding of insulin to its receptors on cardiac cells elicits a series of downstream signal transduction pathways, which results in tight regulation of glucose and fatty acid uptake and utilization, glycogen and protein production, and vascular function (Muniyappa et al. 2007). However, when cells become resistant to the actions of insulin, lipotoxicity (An and Rodrigues 2006) and systemic hyperglycemia ensue; this results in a compensatory increase in insulin secretion (Chavez 2006). As such, persistent hyperinsulinemia resulting from IR can promote dyslipidemia and the proliferation of inflammatory factors (Kasuga 2006), which probably contribute to the onset of overt T2DM and DCM. However, the underlying molecular mechanisms and metabolic changes associated with these abnormalities are largely unknown, but could involve modulation of gene and protein expression.

In diabetic rodent models, DCM is associated with reduced glucose uptake and lipotoxicity, and adverse changes in calcium handling and contractile variables (Belke et al. 2000; Vasanji et al. 2006). In particular, the calcium handling protein sarco(endo)plasmic reticulum $\mathrm{Ca}^{2+}$ ATPase (SERCA), its regulator phospholamban (PLN), and the contractile protein cardiac Troponin I (cTnI), which have pivotal roles in the control of cardiac contraction and relaxation (Bers 2002), are significantly affected in DCM. Many of these studies have found that DCM-like cardiac complications are accompanied by significant changes in the expression and function of these proteins (Frank et al. 2002; Kranias and Bers 2007; Li et al. 2000; Ramirez-Correa and Murphy 2007).

Given the magnitude of the burden of T2DM and DCM, numerous approaches are being explored to prevent and/or reverse the associated metabolic effects. One such approach is in the use of plant-based products, such as anthocyanins, to influence cell signalling mechanisms. Anthocyanins are naturally occurring, pigmented compounds from fruits and vegetables, regularly consumed in fruits and vegetables and represent a major subclass of flavonoids (Mazza and Miniati 1993). Estimates on human intakes vary widely from 3 to $215 \mathrm{mg} /$ day (de Pascual-Teresa et al. 2010). Purple corn anthocyanin has been shown to be protective towards IR, weight gain, elevated serum lipids and the production of tumour necrosis factor- $\alpha$ (TNF- $\alpha$ ) in C57BL/6 rats fed a high-fat diet (Tsuda et al. 2003). Anthocyanins extracted from bilberry effectively improved hyperglycemia and insulin sensitivity in a genetic rodent model of T2DM (KK-A $\mathrm{A}^{\mathrm{y}}$ mice). This was associated with higher levels of AMP-activated protein kinase (AMPK) activity (Takikawa et al. 2010), which is also the target of metformin, a pharmacological agent routinely used in the management of T2DM (Hawley et al. 2002). Given these findings, it appears that anthocyanins can modulate intermediary metabolic pathways and perhaps influence the outcomes of risk factors for T2DM. However, the extent to which anthocyanins are able to reverse or modulate the molecular mechanisms of cardiac IR is unknown, although it is reasonable to assume that changes in the insulin signalling pathway are likely to occur.

We hypothesized that a high-sucrose diet leads to changes in the expression of insulin signalling genes and proteins as well as cardiac contractile proteins, and that these changes can be modulated by treatment with an anthocyanin extract. Our objectives were to reproduce the sucrose-fed insulin-resistant animal model, ascertain changes in gene and protein expression in the insulin signalling pathway in rat hearts and attempt to modulate these changes with an anthocyanin treatment.

\section{Materials and methods}

Bilberry anthocyanin

Commercially available dried bilberry powder, prepared using a low-temperature technique (Natural Newfoundland Nutraceuticals, Newfoundland, Canada) and containing $29.60 \mathrm{mg} \mathrm{GAE} / \mathrm{g}$ total phenolics and $24.05 \mathrm{mg} \mathrm{C} 3 \mathrm{GE} / \mathrm{g}$ anthocyanins was double-extracted with acidified methanol ( $85 \% \mathrm{MeOH}: 14 \% \mathrm{H}_{2} \mathrm{O}: 1 \% \mathrm{HOAc}$ ) and partially purified using flash chromatography (Isolera One, Biotage, Sweden, $\mathrm{AB})$. The latter treatment also resulted in the removal of free sugars. Methanol was removed by rotary evaporation, and the resulting extracts were freeze-dried and stored at $-20{ }^{\circ} \mathrm{C}$ until use. Total anthocyanin concentration (TAC) was determined in triplicate using a modified $\mathrm{pH}$ differential method using a cyanidin-3-glucoside standard (Li et al. 2012).

Bilberry extract was solubilized in $2 \mathrm{~N} \mathrm{HCl}$ solution at $1 \mathrm{mg} / \mathrm{mL}$ and hydrolyzed by incubation in a boiling water bath for $2 \mathrm{~h}$ and further characterized using an UHPLC system (Accela-Thermal Technologies Co., Ltd., USA) equipped with a diode array detector. Separation was achieved with a Phenomenex Kinetex PFP, $4.6 \times 150 \mathrm{~mm}$, a $2.6-\mu \mathrm{m}$ column $(100 \times 2.1 \mathrm{~mm}$, Phenomenex, Torrance, CA, USA) attached to a Phenomenex PFP guard column $(4 \times 3 \mathrm{~mm})$. The column was thermostatically controlled at $30{ }^{\circ} \mathrm{C}$ and the flow rate was set to $1 \mathrm{~mL} / \mathrm{min}$. The mobile phase consisted of water-formic acid (A, 95:5, v/v) and methanol-formic acid (B, 95:5, v/v) with the following 
solvent gradient: $0-25 \mathrm{~min}, 0-50 \% \mathrm{~B} ; 25-27.5 \mathrm{~min}, 50$ $100 \% \mathrm{~B}$, and held at $100 \% \mathrm{~B}$ for additional $2.5 \mathrm{~min}$. The column was then re-equilibrated at $100 \% \mathrm{~A}$ for $5 \mathrm{~min}$. Peak absorbance was collected between 200 and $620 \mathrm{~nm}$ using DAD, and the chromatogram output was set at $520 \mathrm{~nm}$. Stock solutions of the standards were prepared by dissolving $10 \mathrm{mg}$ of each compound in $5 \mathrm{~mL}$ DMSO and then topped up to $100 \mathrm{~mL}$ in a volumetric flask with $50 \%$ methanol (final concentration $100 \mu \mathrm{g} / \mathrm{mL}$ ).

Petunidin was confirmed using a Finnigan LCQ DECA ion trap mass spectrometer (ThermoFnnigan, San Jose, CA, USA) equipped with electrospray ionization (ESI) source. Separation was performed with the same binary solvent system as in the HPLC method, but a different column (Luna 3- $\mu$ m PFP (2) (4.6 mm $\times 100 \mathrm{~mm}$; Phenomenex, Torrance, CA, USA)) and gradient programme: 0-26 min, $10-100 \% \mathrm{~B} ; 26-$ $28.5 \mathrm{~min}, 100-10 \% \mathrm{~B}$, then the gradient was held at $10 \%$ A for an additional $5 \mathrm{~min}$. The flow rate was set at $1.0 \mathrm{~mL} /$ min. Positive mode was selected for data collection. The instrument was tuned by using cyanidin standard to reach its optimum performance. Other analytical conditions have been previously described by (Li et al. 2012).

\section{Experimental design}

Male Sprague Dawley rats, approximately $200 \mathrm{~g}$ and 6 weeks of age, were randomly divided into three experimental groups (four rats/cage): control (C), sucrose-fed (SF) and sucrose-fed anthocyanin treated (SF-A) as shown in Table 1. Control animals were fed a basal AIN-93 diet while the SF and SF-A animals were fed a high-sucrose diet. The diets were isocaloric; however, the high-sucrose diet contained $55 \%$ of energy as sucrose, whereas in the basal diet, energy was mainly from cornstarch. All diets were formulated by Dyets Incorporated (PA, USA), and the compositions are shown in Table 1.
The animals were all fed the control diet for 3 days, after which a tail vein blood sample was taken for baseline measurements. All the groups were fed and watered ad libitum. Anthocyanin was supplemented (SF-A) by addition to drinking water $(0.2 \mathrm{mg} / \mathrm{mL}$ anthocyanin) which was stored in dark bottles and replaced every 2 days. Chow intake and body weight were measured weekly and fluid intake every 2 days. Based on the weekly chow intake and the energy content of the diet (Table 1), the average energy intake per rat for each week was calculated and standardized according to body weights. At 3-week intervals, the animals were fasted overnight and $\sim 1.0 \mathrm{~mL}$ blood was drawn into freshly prepared heparinized tubes ( 3 units $/ 0.5 \mathrm{~mL}$ blood) using the tail clip method. Plasma was separated and stored at $-70{ }^{\circ} \mathrm{C}$ pending batch analyses for glucose, insulin and triglycerides. After 14 weeks, an intraperitoneal glucose tolerance test (IPGTT) was performed on the fasted animals by intraperitoneal injection of a glucose solution ( $2 \mathrm{~g} / \mathrm{kg}$ body weight). Blood was then drawn and analysed for glucose using a Hemocue 201 Analyzer (Hemocue AB, Ängelholm, Sweden) at baseline and at 30, 60 and $120 \mathrm{~min}$ following glucose administration. One week after the IPGTT, the animals were terminally anaesthetized; the heart was harvested, flash-frozen in liquid nitrogen and stored at $-80{ }^{\circ} \mathrm{C}$ pending biochemical analyses.

\section{Biochemical analyses}

Commercially available analytical kits were used to measure plasma levels of triglycerides (Human Diagnostics, Wiesbaden, Germany) and insulin (Linco Research, MO, USA). The oxygen radical absorbance capacity (ORAC) (Prior et al. 2003) and ferric reducing antioxidant power (FRAP) (Benzie and Strain 1996) assays were used to determine total antioxidant capacity of serum. The homeostasis assessment model (HOMA) was used to

Table 1 Experimental treatments and diet composition

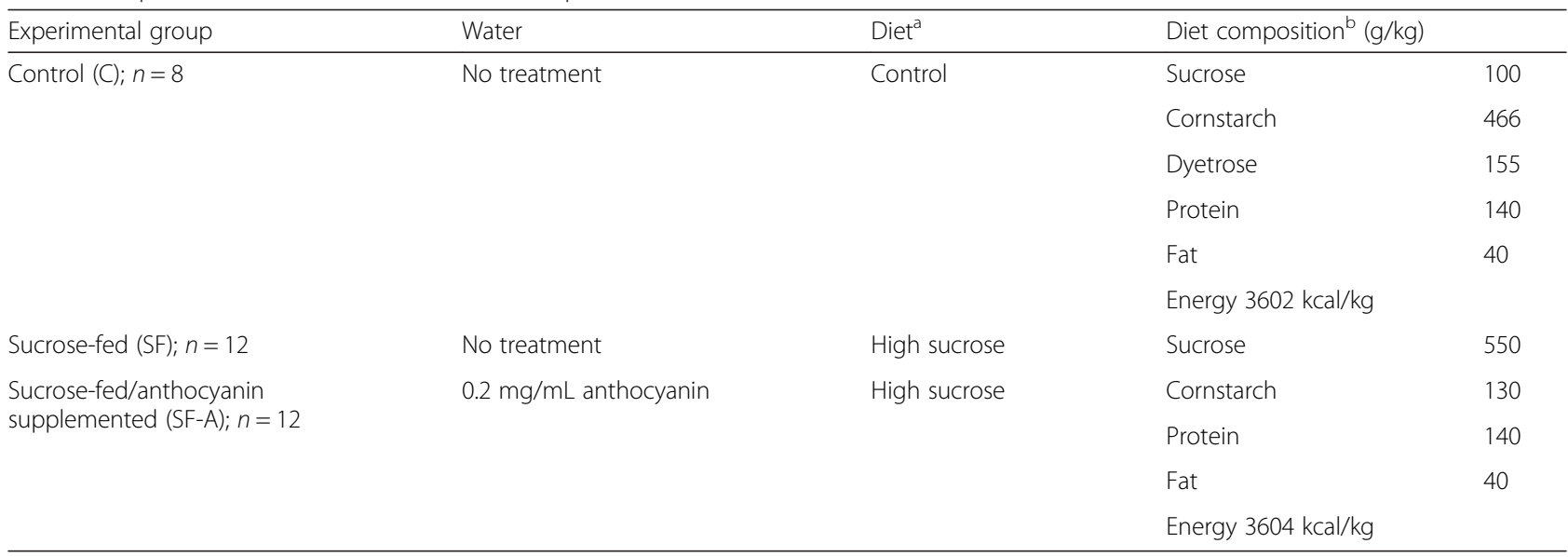

aiets were formulated by Dyets Incorporated, PA, USA

${ }^{\mathrm{b}}$ Both diets contained the recommended concentrations of vitamins, minerals and fibre 
calculate HOMA-IR. This was calculated by multiplying fasting plasma insulin (FPI) by fasting plasma glucose (FPG), then dividing by 22.5 (Diabetes Trials Unit, University of Oxford, UK).

For gene expression studies, RNA was isolated from heart tissue using TRI Reagent (Sigma-Aldrich, CA, USA). Impurities were removed using RNeasy Mini Kit (Qiagen, Valencia, CA), and RNA quality was assessed using the absorbance ratios at $260 \mathrm{~nm} / 230 \mathrm{~nm}$ and $260 \mathrm{~nm} / 280 \mathrm{~nm}$. Purified RNA was then used to quantify gene expression for 84 genes along the insulin signalling pathway utilizing the 7500 ABI Fast Real Time PCR System (Applied Biosystems, CA, USA) with the $\mathrm{RT}^{2}$ Profiler PCR Array System (SABiosciences, Frederick, MD, USA). $C_{\mathrm{t}}$ (cycle threshold) values were generated by the 7500 ABI Fast Real Time PCR System and used to calculate fold changes in gene expression. Fold change differences between experimental groups were calculated for each gene as follows: Fold $\Delta(\mathrm{GOI})=$ Ave $\left[C_{\mathrm{t}}(\mathrm{TEST})-C_{\mathrm{t}}(\mathrm{HKG})\right] /$ Ave $\left[C_{\mathrm{t}}(\mathrm{CONTROL})-C_{\mathrm{t}}(\mathrm{HKG})\right]$, where GOI is gene of interest and HKG is housekeeping gene.

Proteins were prepared by dicing and homogenizing left ventricular tissue with a hand-held homogenizer in cold lysis buffer: $50 \mathrm{mM}$ Tris- $\mathrm{HCl} \mathrm{pH}$ 7.5, $150 \mathrm{mM} \mathrm{NaCl}, 0.1 \%$ SDS, 1 mM EGTA, containing protease inhibitors (1:250) (Roche Diagnostics Corporation, IN, USA), phosphatase inhibitor cocktails I and II $(1 \mathrm{~mL} / 100 \mathrm{~mL}$ buffer, SigmaAldrich, CA, USA) and protein kinase inhibitor $\mathrm{H}-7(1 \mathrm{~mL} /$ $100 \mathrm{~mL}$ buffer, Sigma-Aldrich, CA, USA). This homogenate was then centrifuged at $10,000 \times g$ for $10 \mathrm{~min}$ at $4{ }^{\circ} \mathrm{C}$. The supernatant was removed, aliquoted and stored at $-70{ }^{\circ} \mathrm{C}$ pending sodium dodecyl sulphate polyacrylamide gel electrophoresis (SDS-PAGE). Proteins were electrophoresed on $12 \%$ polyacrylamide gels at $150 \mathrm{~V}$ for $1.5 \mathrm{hr}$ under denaturing conditions (Laemmli 1970) and subsequently transblotted onto polyvinyl difluoride membrane (Towbin et al. 1979) and probed with antibodies for PLN, SERCA, cTnI, the insulin receptor, Akt, AMPK and the phosphorylated forms of these proteins (Badrilla, Leeds, UK; Affinity Bioreagents, CO, USA; Cell Signalling Technology MA, USA; Abcam, MA, USA). Primary antibodies were diluted at a ratio of 1:1000, and secondary antibodies were diluted at a ratio of 1:2000. Blots were then quantified using Image J (NIH, MD, USA) and normalized using the protein glyceraldehyde-3-phosphate dehydrogenase (GAPDH) (Cell Signalling Technology MA, USA). The protein expression ratios of PLN/SERCA and phosphorylated to unphosphorylated protein for cTnI, the insulin receptor, Akt and AMPK were calculated and compared between experimental groups to gauge the functionality of these proteins.

\section{Statistical analyses}

The study was designed using a power analysis based on an expected difference of $50 \%$ in the area under the glucose response curve following an IPGTT, a type I error of 0.05 and a power of $90 \%$. The required sample size was calculated to be at least eight. Statistical analyses were performed using the SPSS 12 software for Windows (SPSS Inc., Chicago, IL, USA). Differences in insulin, glucose and triglyceride levels and blood glucose during the IPGTT, between control and SF animals, were evaluated using repeated measures ANOVA. The differences in ORAC, FRAP, $C_{\mathrm{t}}$ values, AUC from the IPGTT and western blotting results were compared using one way ANOVA. A $p$ value of less than 0.05 was regarded as being significant.

\section{Results}

The bilberry extract had a total anthocyanin content of $204.24 \pm 2.17 \mathrm{mg}$ CyGE/100 g DW and was consistent with other studies (Bakowska-Barczak et al. 2007; Zhang et al. 2004), the major anthocyanidin aglycones being delphinidin, cyanidin, petunidin, peonidin and malvidin (Fig. 1). Among the study groups, there were no significant differences in body weight (Fig. 2) or energy consumption (Fig. 3). After 15 weeks on the experimental diet, neither HOMA-IR (Fig. 4) nor plasma triglyceride concentration (Fig. 5) was significantly different among the study groups at any time point. The IPGTT also showed no significant differences among the groups for blood glucose levels at any time point or in the incremental area under the blood glucose response curve (Fig. 6). The FRAP assay showed significantly lower values for SF when compared to control, and significantly higher values for SF-A when compared to SF animals (Fig. 7a). However, ORAC values were not significantly different among the experimental groups (Fig. 7b).

When SF treatment was compared to control, PCR array gene analyses of left ventricular tissues showed that the expression of several genes were significantly down-regulated $(p<0.05$; Table 2$)$ and included acetylcoenzyme A carboxylase alpha (ACC1), V-Akt murine thymoma viral oncogene homolog 1 (Akt), Bcl2-like 1 (Bcl2), CCAAT/enhancer binding protein $(\mathrm{C} / \mathrm{EBP})$, beta $(\mathrm{C} / \mathrm{EBP} \beta)$, FK506 binding protein 12-rapamycin associated (mTOR), glycerol-3-phosphate dehydrogenase 1 (soluble) (GPDH1), hexokinase 2 (HK2), solute carrier family 2 (facilitated glucose transporter), member 1 (GLUT1), solute carrier family 2 (facilitated glucose transporter), member 4 (GLUT4) and Thyroglobulin (Tg). These changes were not observed when SF-A animals were compared with control, indicating that anthocyanin supplementation prevented these changed in gene expression. Further, when SF was compared with SF-A treatment, complement factor D (adipsin) and phosphoinositide-3-kinase, regulatory subunit $1(\alpha)$ $(\mathrm{PI} 3 \mathrm{~K} \alpha)$ were significantly up-regulated $(p<0.05)$, while sterol regulatory element binding transcription factor 1 


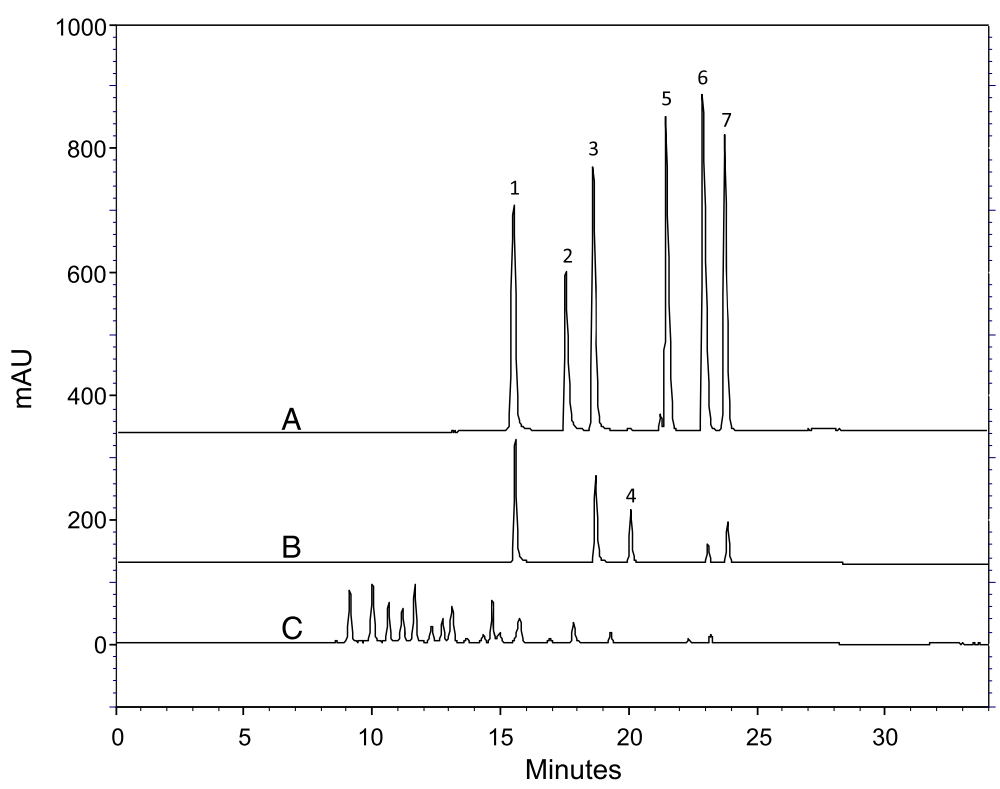

Fig. 1 UHPLC-DAD chromatograms of mixed standards (A) and bilberry extract before $(C)$ and after acid hydrolysis (B). Mobile phase A—95\%:5\% water-formic acid; mobile phase B—95 \% methanol:5\% formic acid $(\mathrm{v} / \mathrm{v})$; flow rate $1.0 \mathrm{~mL} / \mathrm{min}$; column: Phenomenex Kinetex PFP, $4.6 \times 150 \mathrm{~mm}$, $2.6 \mu \mathrm{m}$; detection at $520 \mathrm{~nm}$; injection: $10 \mu \mathrm{L}$. A = standards: 1 delphinidin, 2 luteolinidin, 3 cyanidin, 4 petunidin, 5 pelargonidin, 6 peonidin, 7 malvidin; $B=$ hydrolyzed bilberry extract; $C=$ bilberry extract

(SREBF1) was significantly down-regulated $(p<0.05)$ (Table 3).

Compared to control, PLN levels were significantly increased $(p<0.05)$ and $\mathrm{p}^{*}$-cTnI levels were significantly decreased $(p<0.05)$ in the heart of SF-treated rats (Fig. 8a, d, respectively); these changes were not attenuated by anthocyanin supplementation. However, total AMPK expression was significantly lower $(p<0.05)$ in SF-A when compared to both control and SF hearts (Fig. 8i), while protein expression for SERCA, cTnI, IRec, $\mathrm{p}^{*}$-IRec, Akt, $\mathrm{p}^{*}$-Akt and $\mathrm{p}^{*}$-AMPK were not different (Fig. 8b, c, e-h, j, respectively). The ratio of SERCA/PLN was significantly higher

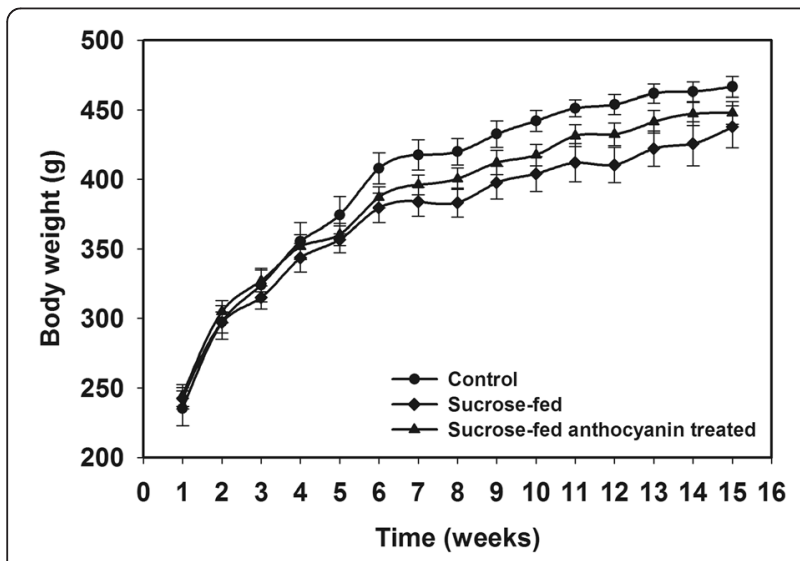

Fig. 2 Body weights (mean \pm SE) for control $(n=8)$, sucrose-fed $(n=12)$ and sucrose-fed anthocyanin treated $(n=12)$ rats over 15 weeks $(p<0.05)$ in SF-A rats when compared to that in SF (Fig. 9a), whereas the ratio of $\mathrm{p}^{*}-\mathrm{cTnI} / \mathrm{cTnI}$ was significantly decreased in both SF and SF-A animals (Fig. 9b).

\section{Discussion}

We found that feeding male Sprague Dawley rats a solid high-sucrose diet for 15 weeks did not result in the animals becoming insulin resistant, hypertriglyceridemic or obese, although others have been able to achieve these metabolic abnormities using sucrose diets (Davidoff et al. 2004; Vasanji et al. 2006). In our laboratory, when these animals are fed sucrose $(32 \%, w / v)$ in drinking

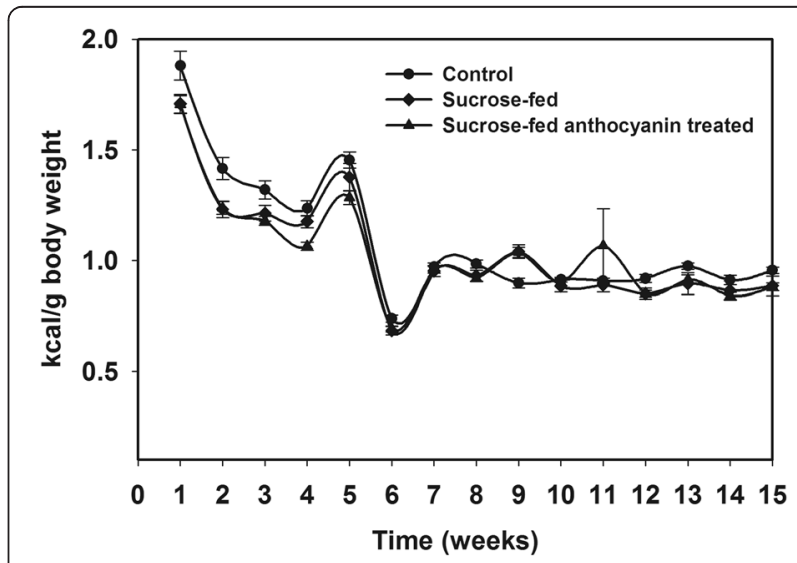

Fig. 3 Energy consumption in $\mathrm{kcal} / \mathrm{g}$ body weight (mean \pm SE) for control $(n=8)$, sucrose-fed $(n=12)$ and sucrose-fed anthocyanin treated $(n=12)$ rats over 15 weeks 


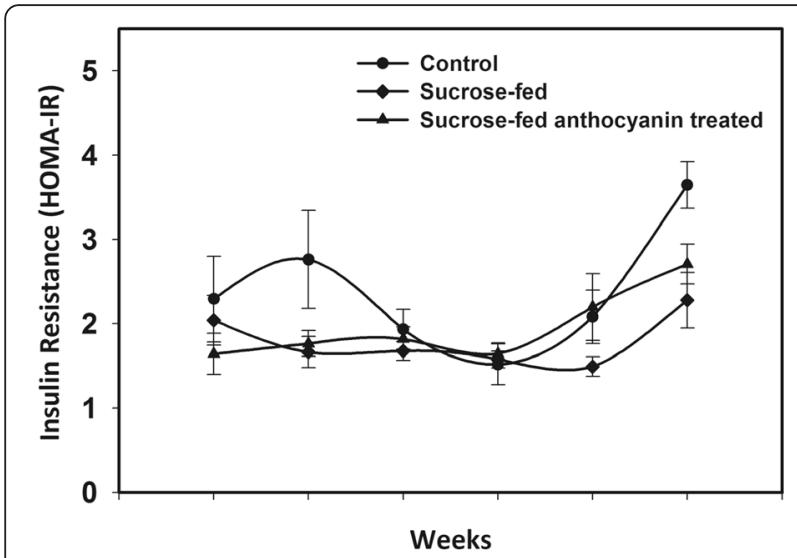

Fig. $4 \mathrm{HOMA}-\mathrm{IR}$ (mean \pm SE) for control $(n=8)$, sucrose-fed $(n=12)$ and sucrose-fed anthocyanin treated $(n=12)$ rats over 15 weeks

water (presumably absorbed primarily in the small intestine), they become obese and insulin resistant after 810 weeks of feeding. However, they consume less solid food and increase water consumption, so that their nutrient intake profile becomes altered (Singh and Ramdath unpublished); this would be a concern in efforts to establish a diet-induced rodent model of T2DM. Recent studies have suggested that the gut microbiota might play a crucial role in the onset of diabetes (Everard and Cani 2013; Turnbaugh et al. 2008). As such, it is possible that our animals, obtained from the local animal house, might have acquired a microbiota that is different from animals obtained from a central breeding source, and this could account for their resistance to developing a diabetic/prediabetic condition when fed orally with a solid high-sucrose diet. Others have shown that both Sprague Dawley and Wistar rats failed to become obese on a high-fat diet (Perez de Heredia et al. 2006) and may have different rates of weight gain which is associated with hyperglycaemia (Lauterio et al. 1994). It seems reasonable to expect that

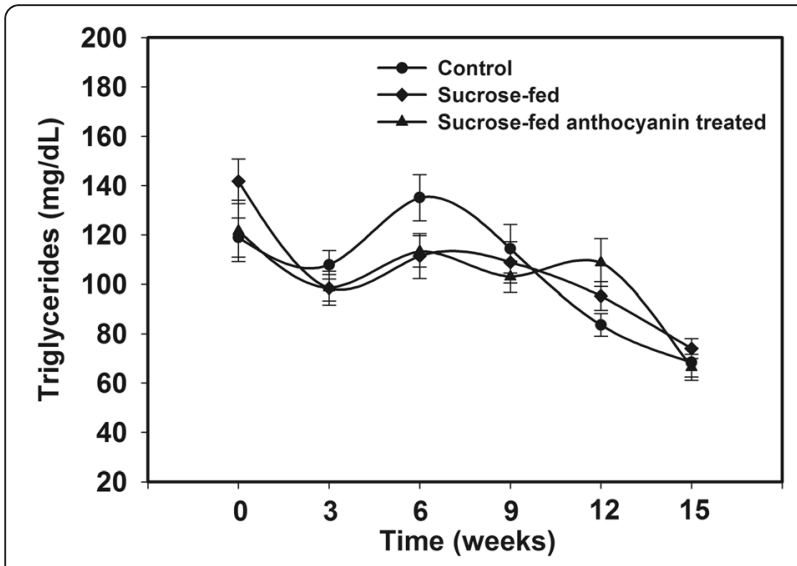

Fig. 5 Plasma triglycerides (mean \pm SE) for control $(n=8)$, sucrose-fed $(n=12)$ and sucrose-fed anthocyanin treated $(n=12)$ rats over 15 weeks

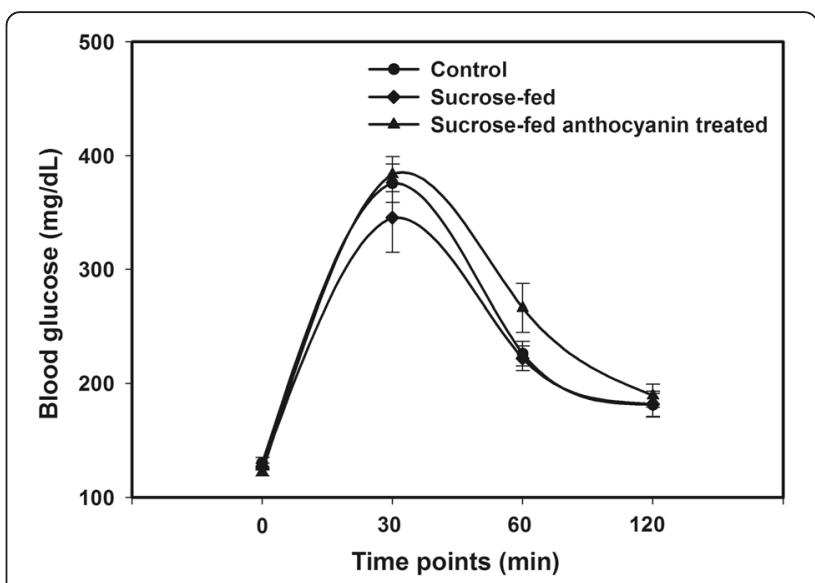

Fig. $6 \mathrm{IPGTT}$ test (mean \pm SE) for control $(n=8)$, sucrose-fed $(n=12)$ and sucrose-fed anthocyanin treated $(n=12)$ rats after 15 weeks

there is inherent metabolic variation (metabolic plasticity) among rodents that allows them to resist changes in glucose and lipid metabolism (Chang et al. 1990).

Clearly, the fact that our animals showed no abnormal metabolic changes or signs of DCM on a high-sucrose diet is not unique. We contend that our results from gene and protein expression assays are indicative of very early cellular changes in insulin signalling and cardiac function resulting from a high-sucrose diet. It is likely that these will eventually lead to phenotypic changes and result in overt metabolic changes. However, the length of time between these events is likely to be influenced by cellular adaptation and metabolic plasticity, as mentioned above. Moreover, identification of very early changes in the expression of genes and proteins associated with insulin signalling and cardiac function could allow for a better understanding of DCM aetiology and facilitate early intervention.

Using the multiple gene profiling feature of microarray analysis with real-time PCR quantitation techniques, we showed that expression of GLUT1, GLUT4, HK2, GLUT4 and Akt were down-regulated. Decreased expression of GLUT1 and GLUT4 may be indicative of reduced glucose transport into cardiomyocytes, which could impair cardiac muscle contractility (Abel 2004). However, in mice with cardiac-specific inactivation of the GLUT4 gene, there is modest ventricular hypertrophy with no contractile changes (Abel et al. 1999). In contrast, decreased expression of HK2 in sucrose-fed rats, as found on our study, has been linked to a reduction glucose phosphorylation and possibly a reduction in ATP production and contractility (Ye et al. 2005). Further, cardiac contractility might have been slightly impaired in our SF animals, given the small but significant decrease in Akt gene expression observed. In a similar study, Roberts et al. 2005 showed that a high-fat, highsugar diet resulted in decreased Akt protein expression 

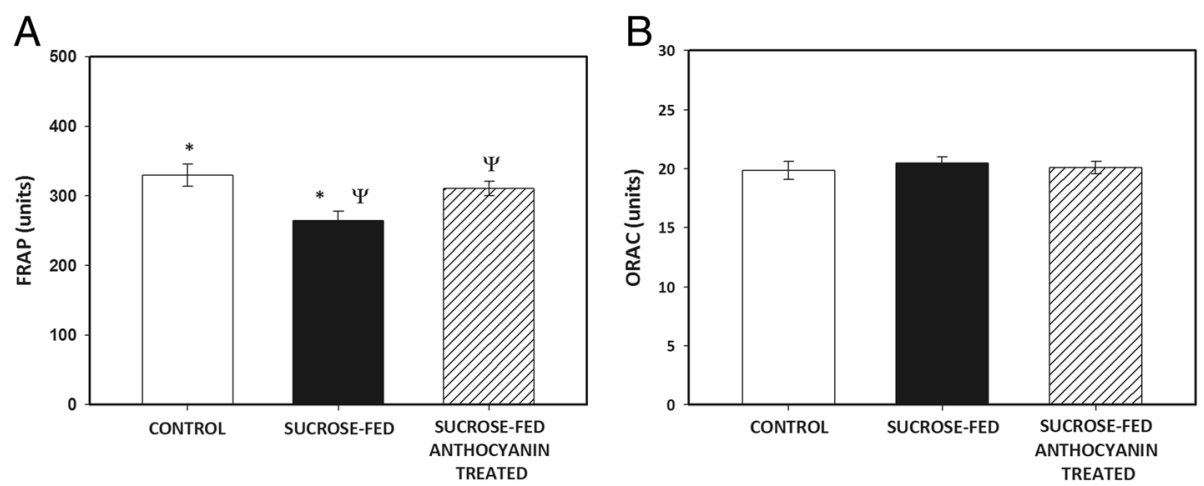

Fig. 7 a Ferric reducing antioxidant power (FRAP) values (mean \pm SE) for control $(n=8)$, sucrose-fed $(n=12)$ and sucrose-fed anthocyanin treated $(n=12)$ rats after 15 weeks. ${ }^{*}, \Psi$ significantly different at $\left.p<0.05\right)$. b Oxygen radical absorbance capacity $($ ORAC) values (mean \pm SE) for control $(n=8)$, sucrose-fed $(n=12)$ and sucrose-fed anthocyanin treated $(n=12)$ rats after 15 weeks (1 ORAC unit equals to the net antioxidant protection produced by $1 \mu \mathrm{M}$ Trolox (the water-soluble vitamin E analogue used as a positive control))

in aorta and heart tissue; they suggested that eNOS activation by Akt and therefore endothelial function may be impaired by HFS. Akt phosphorylates a wide range of substrates in the proximal part of the insulin signalling pathway, and impairment of this function has been shown to result in downstream contractile abnormalities, similar to those associated with IR and DCM. However, the ratio of p-Akt/Akt, which is the activation status of Akt, was unaltered, suggesting that sucrose feeding did not affect Akt activation. As such, the metabolic effects of decreased Akt protein expression require further investigation (Roberts et al. 2005).

Taken together, changes in the expression of genes along the insulin signalling pathway as well as those involved in cellular energy utilization (ACC1, mTOR and GPDH1) suggest that glucose transport and usage is decreased in the heart of SF-fed rats, and this may result in impaired cardiac contractility. This is consistent with observations in diabetic rats: they exhibit cardiac dysfunction and IR associated with decreased glucose and increased fatty acid utilization, decreased mRNA and protein levels of GLUT4 (van den Brom et al. 2009). Furthermore, defects in GLUT4 translocation has been demonstrated in the skeletal muscle of Goto-Kakizaki rats and in type 2 diabetic patients (Zdychova and Komers 2005). Collectively, reductions in the expression of $\mathrm{C} / \mathrm{EBP} \beta$ and $\mathrm{Bcl} 2$ and genes that play a critical role in cellular energy homeostasis may provide an aetiological mechanism for the onset of DCM following high-sucrose feeding (Bosch et al. 1995; Cory and Adams 2002; Hage et al. 2011).

Our protein expression data further suggests that high-sucrose feeding might have resulted in impaired cardiac contractility. We found that SF led to increase levels of PLN, while SERCA/PLN ratio, $\mathrm{p}^{*}$-cTnI and $\mathrm{p}^{*} \mathrm{cTnI} / \mathrm{cTnI}$ ratio were decreased. An increase in PLN is likely to increase inhibition of SERCA and prolong

Table 2 Mean $( \pm S E) C_{t}$ values for cardiac genes that showed different expression levels when SF samples were compared to control samples

\begin{tabular}{|c|c|c|c|c|}
\hline Description & $\begin{array}{l}\text { Control } \\
(n=5)\end{array}$ & $\begin{array}{l}\text { Sucrose-fed } \\
(n=5)\end{array}$ & $p$ value & ${ }^{\mathrm{a}}$ Fold $\Delta$ \\
\hline Acetyl-coenzyme A carboxylase alpha & $26.71 \pm 0.13$ & $27.21 \pm 0.16$ & 0.038 & -1.12 \\
\hline V-Akt murine thymoma viral oncogene homolog 1 & $25.65 \pm 0.10$ & $26.03 \pm 0.11$ & 0.027 & -1.04 \\
\hline Bcl2-like 1 & $23.61 \pm 0.12$ & $24.20 \pm 0.22$ & 0.047 & -1.19 \\
\hline CCAAT/enhancer binding protein (C/EBP), beta & $23.45 \pm 0.16$ & $24.14 \pm 0.23$ & 0.037 & -1.28 \\
\hline MTOR- FK506 binding protein 12-rapamycin associated & $25.08 \pm 0.09$ & $25.60 \pm 0.17$ & 0.026 & -1.13 \\
\hline Glycerol-3-phosphate dehydrogenase 1 (soluble) & $24.61 \pm 0.24$ & $25.42 \pm 0.14$ & 0.019 & -1.38 \\
\hline Hexokinase 2 & $26.37 \pm 0.13$ & $27.25 \pm 0.29$ & 0.022 & -1.46 \\
\hline Solute carrier family 2 (facilitated glucose transporter), member 1 & $29.45 \pm 0.19$ & $30.49 \pm 0.36$ & 0.034 & -1.64 \\
\hline Solute carrier family 2 (facilitated glucose transporter), member 4 & $23.21 \pm 0.09$ & $24.07 \pm 0.20$ & 0.005 & -1.44 \\
\hline Thyroglobulin & $29.88 \pm 0.18$ & $30.40 \pm 0.09$ & 0.037 & -1.12 \\
\hline
\end{tabular}

All were significant at $p<0.05$

- represent downregulation

${ }^{\mathrm{a}}$ Fold changes; calculated after $C_{\mathrm{t}}$ values were normalized using housekeeping genes 
Table 3 Mean $( \pm S E) C_{t}$ values for cardiac genes that showed different expression levels when SF-A samples were compared to SF samples

\begin{tabular}{llrr}
\hline Description & $\begin{array}{l}\text { Sucrose-fed } \\
(n=5)\end{array}$ & $\begin{array}{l}\text { Sucrose-fed anthocyanin treated } \\
(n=5)\end{array}$ & $p$ value \\
\hline Complement factor D (adipsin) & $26.05 \pm 0.29$ & $25.31 \pm 0.13$ & 0.045 \\
Phosphoinositide-3-kinase, regulatory subunit 1 (alpha) & $24.23 \pm 0.19$ & $23.62 \pm 0.14$ & 0.030 \\
Sterol regulatory element binding transcription factor 1 & $34.25 \pm 0.21$ & $34.82 \pm 0.15$ & +1.48 \\
\hline
\end{tabular}

All were significant at $p<0.05$

- represents downregulation, + represent upregulation

${ }^{a}$ Fold changes; calculated after $C_{\mathrm{t}}$ values were normalized using housekeeping genes
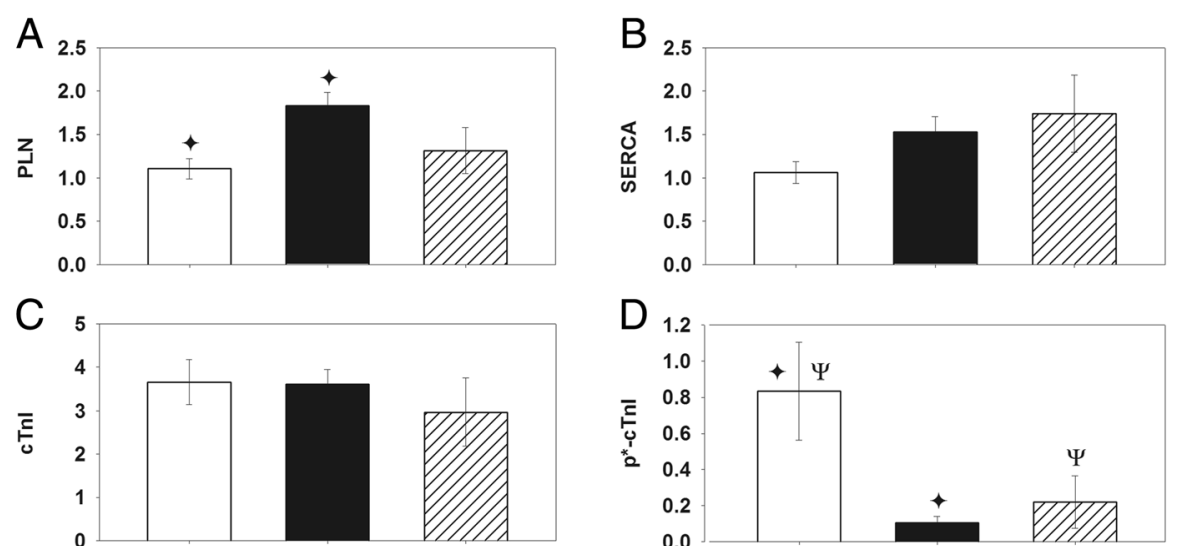

$D_{1.2}$

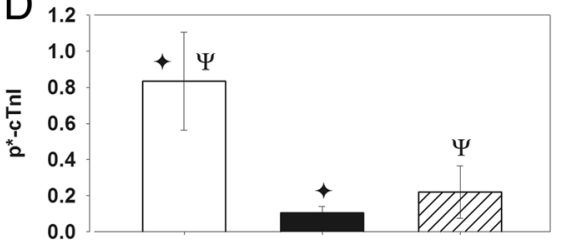

E

$\mathrm{F}$

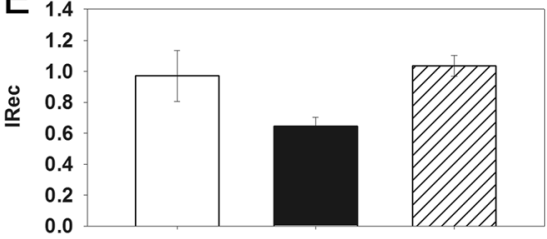

$\mathrm{G}$

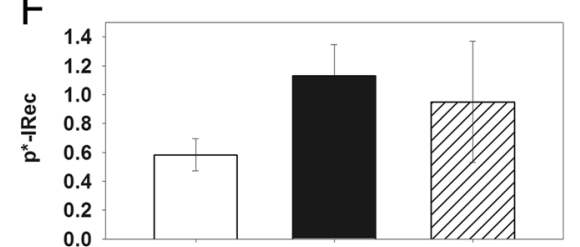

$\mathrm{H}$
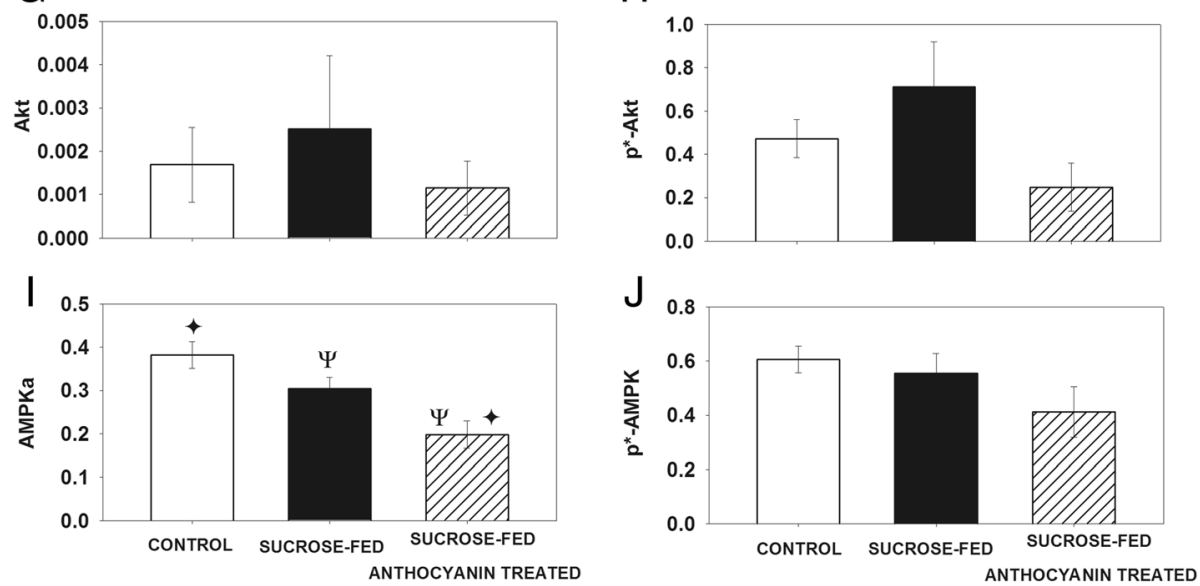

Fig. 8 Protein expression for PLN (a), SERCA (b), CTnl (c), $p^{*}-C T n l(\mathbf{d})$, IRec (e), $p^{*}-I \operatorname{Rec}(\mathbf{f})$, Akt (g), $p^{*}$-Akt (h), AMPK (i) and p*-AMPK (j) in control, sucrose-fed and sucrose-fed anthocyanin treated rat heart homogenates. All densitometric measurements were normalized using GAPDH before comparisons were made ( $p^{*}$ phosphorylated; $\psi$ significantly different at $p<0.05 ; n=5$ ) 

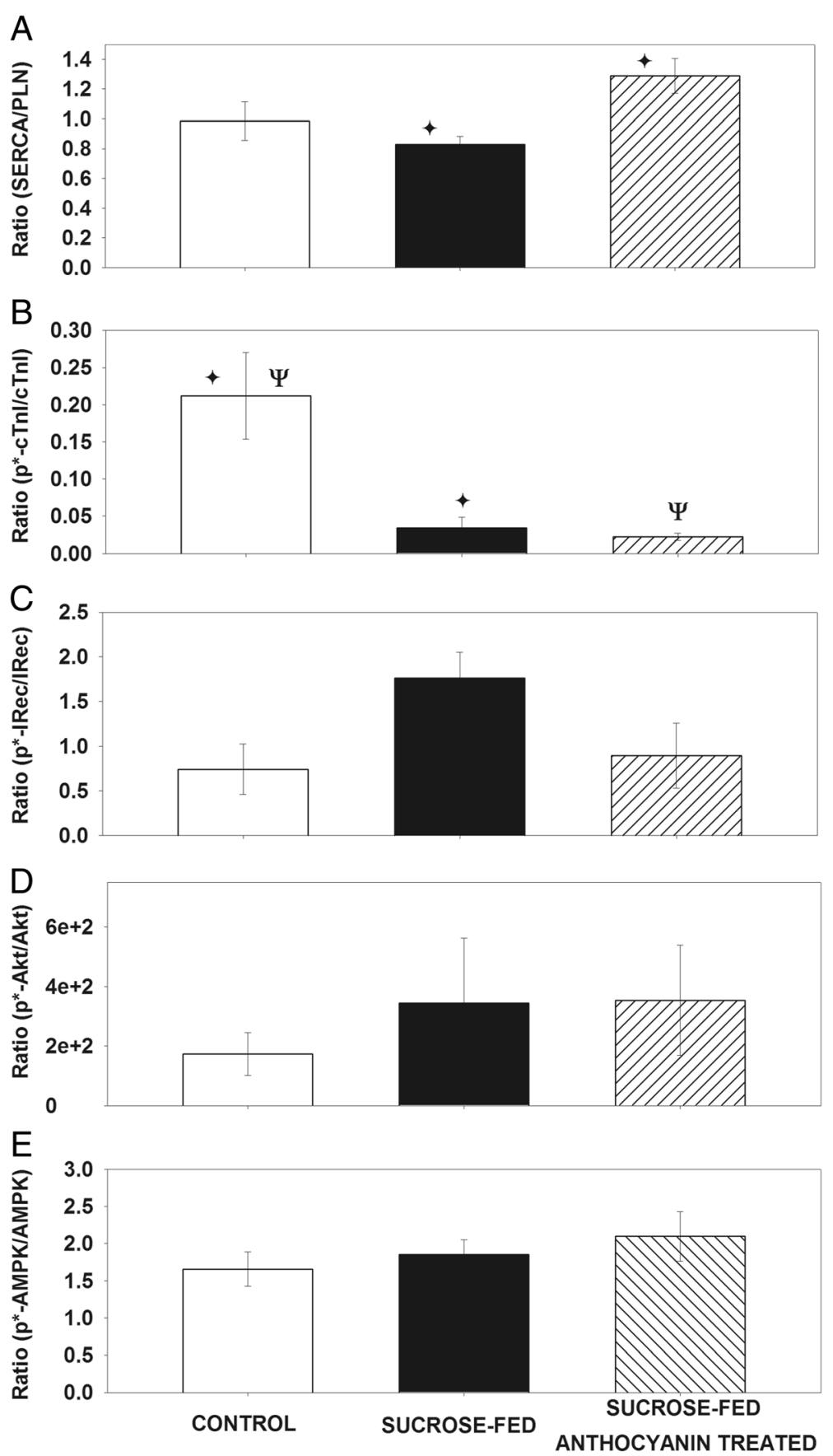

Fig. 9 Ratios of SERCA/PLN (a), $p^{*}-c T n l / c T n l(\mathbf{b}), p^{*}-I \operatorname{Rec} / \operatorname{Rec}(\mathbf{c}), \mathrm{p}^{*}$-Akt/Akt (d) and $\mathrm{p}^{*}$-AMPK/AMPK (e) in control, sucrose-fed and sucrose-fed anthocyanin treated rat heart homogenates ( $p^{*}$ phosphorylated; $\psi \psi$ significantly different at $p<0.05 ; n=5$ )

relaxation rates of cardiac muscles (Koss et al. 1997); decreased $\mathrm{p}^{*}$-cTnI and $\mathrm{p}^{*} \mathrm{cTnI} / \mathrm{cTnI}$ ratio could also contribute to lengthen rates of relaxation via a depression of cross-bridge cycling (Zhang et al. 1995). An analysis of the expression and activity of PKA, CaMKII and the other Tn subunits may be required in order to fully interpret these results. AMPK is regarded as a metabolic sensor since it upregulates catabolic pathways that generate ATP and down-regulates anabolic pathways that consume ATP (Hardie 2008). In the present study, expression of AMPK protein was decreased with sucrose feeding. However, the ratio of p-AMPK/AMPK, which is the activation status of AMPK was unaltered, suggesting that sucrose feeding did not affect AMPK activation.

Anthocyanin supplementation of the high-sucrose diet augmented the changes in gene expression induced by 
the SF diet. SF-A further led to an increased expression of adipsin and PI3K $\alpha$ and decreased the expression of SREBF1. PI3K $\alpha$ has a central role in the proximal part of the insulin signalling pathway and increased expression may represent a compensatory mechanism to decreased expression of GLUT1, GLUT2 and HK2. SREBF1 expression, shown to be reduced in T2DM (Sewter et al. 2002), represents a series of transcription factors that responds to both Akt and MAPK pathways and influences lipogenesis and TAG deposition in skeletal muscle (Lecomte et al. 2010). As such, downregulation of SREBF1 by anthocyanin supplementation could result in the prevention of lipid accumulation in the heart. Indeed, anthocyanins have been shown to prevent the onset of obesity in animal models (Lee et al. 2014; Wu et al. 2013). An increase in ratio of SERCA/PLN, as seen in SF-A rats, has been shown to promote contractile efficiency (Currie and Smith 1999), and therefore supplementation with anthocyanins may improve heart function.

In our study, we used both ORAC and FRAP assays to estimate the antioxidant potential of serum and found that ORAC was not different among treatment groups, but FRAP was decreased with SF, and SF-A prevented this decrease. These findings are likely due to the fact that FRAP and ORAC assess different antioxidant potentials. ORAC measures the ability of antioxidants in a sample to quench one type of free radical (probably peroxyl) and is influenced by material with and without lag phases of their antioxidants (Cao and Prior 1998). On the other hand, FRAP measures the reducing power of plasma and has been shown to increase with polyphenol supplementation (Benzie et al. 1999). Others have found that sucrose feeding results in depressed level of antioxidants (Diniz et al. 2008; Fang et al. 2005), possibly resulting from oxidative stress which is associated with disrupted insulin signalling and obesity (Styskal et al. 2012). The bilberry anthocyanin supplement used in our study provided a rich source of polyphenols which would likely account for the increase in FRAP, but not ORAC.

In the present study, feeding Sprague Dawley rats a high-sucrose diet did not result in IR. However, the collective evidence from gene expression and protein quantitation studies suggest that the animals experience very early cellular changes in insulin signalling and cardiac function, which may be some of the earliest cellular adaptations to a high-sucrose diet. Importantly, anthocyanins appear capable of modulating some of these changes and may represent a possible therapeutic adjunct in the management of T2DM.

\section{Compliance with ethics guidelines}

All applicable international, national and/or institutional guidelines for the care and use of animals were followed.
All procedures performed in the studies involving animals were in accordance with the ethical standards of the institution or practice at which the studies were conducted. Approval was granted for the use of animals from the Ethics Committee, Faculty of Medical Sciences, The University of the West Indies, St. Augustine, Trinidad and Tobago.

\section{Abbreviations}

ACC1: acetyl-coenzyme A carboxylase alpha; Akt: V-Akt murine thymoma viral oncogene homolog 1; AMPK: 5'-AMP-activated protein kinase; BAcn: bilberry anthocyanin; BCl2: BCl2-like 1; CCAAT/EBP: cytosine-cytosine-adenosineadenosine-thymidine/enhancer binding protein; cTnl: cardiac troponin l; FRAP: ferric reducing antioxidant power; GLUT1: solute carrier family 2 (facilitated glucose transporter), member 1; GLUT4: solute carrier family 2 (facilitated glucose transporter), member 4; GPDH1: glycerol-3-phosphate dehydrogenase 1 (soluble); HK2: hexokinase 2; HOMA-IR: homeostasis model assessment-estimated insulin resistance; IPGTT: intraperitoneal glucose tolerance test; IR: insulin resistance; mTOR: FK506 binding protein 12-rapamycin associated; ORAC: oxygen radical absorbance capacity; $\mathrm{p}^{*}$ : phosphorylated; PI3Ka: phosphoinositide-3-kinase, regulatory subunit 1 (a); PLN: phospholamban, SREBF1, sterol regulatory element binding transcription factor 1; RT-qPCR: reverse transcription quantitative real-time polymerase chain reaction; SERCA: sarco(endo)plasmic reticulum $\mathrm{Ca}^{2+}$ ATPase; SF: sucrose-fed; SF-A: sucrose-fed + BAcn; Tg: thyroglobulin; T2DM: type 2 diabetes mellitus.

\section{Competing interests}

The authors declare that they have no competing interests.

\section{Author's contributions}

DDR and SS conceptualized the study and obtained funding. SS conducted the experiments, cleaned the data and performed initial statistical analysis and reporting. TN supervised the identification of cardiac contractile proteins. All authors interpreted the data and contributed to the final manuscript.

\section{Acknowledgements}

This work was supported in part by the A-base research programme of Agriculture \& Agri-Food Canada (RBPI \#1343) and The University of the West Indies, St. Augustine, Trinidad and Tobago (CRP.5.NOV08.5). We thank Aileen Hawke and Dr. Rong Tsao for the assistance in preparing the anthocyanin extract from bilberry and Dr. Ronghua Liu for the assistance with the polyphenol, anthocyanin and antioxidant analyses.

\section{Author details}

${ }^{1}$ Department of Pre-Clinical Sciences, Faculty of Medical Sciences, The University of the West Indies, St. Augustine Campus, St. Augustine, Trinidad and Tobago, West Indies. ${ }^{2}$ Canadian Centre for Agri-Food Research in Health and Medicine, 351 Taché Avenue, Winnipeg, Manitoba, Canada. ${ }^{3}$ Guelph Food Research Centre, Agriculture and Agri-Food Canada, 93 Stone Road West, Guelph, ON N1G 5C9, Canada.

Received: 29 March 2015 Accepted: 9 January 2016

Published online: 17 March 2016

\section{References}

Abdul-Ghani MA, DeFronzo RA (2009) Plasma glucose concentration and prediction of future risk of type 2 diabetes. Diabetes Care 32 Supple 2:S194-S198. doi:10.2337/dc09-S309

Abel ED (2004) Glucose transport in the heart. Front Basic 9:201-215

Abel ED et al (1999) Cardiac hypertrophy with preserved contractile function after selective deletion of GLUT4 from the heart. J Clin Invest 104:1703-1714. doi:10.1172/JC17605

An D, Rodrigues B (2006) Role of changes in cardiac metabolism in development of diabetic cardiomyopathy. Am J Physiol Heart Circ Physiol 291:H1489-H1506. doi:10.1152/ajpheart.00278.2006

Asghar O et al (2009) Diabetic cardiomyopathy. Clin Sci (Lond) 116:741-760. doi:10.1042/CS20080500 
Bakowska-Barczak AM, Marianchuk M, Kolodziejczyk P (2007) Survey of bioactive components in Western Canadian berries. Can J Physiol Pharmacol 85:1139-1152. doi:10.1139/Y07-102

Belke DD, Larsen TS, Gibbs EM, Severson DL (2000) Altered metabolism causes cardiac dysfunction in perfused hearts from diabetic $(\mathrm{db} / \mathrm{db})$ mice. Am J Physiol Endocrinol Metab 279:E1104-E1113

Benzie IF, Strain JJ (1996) The ferric reducing ability of plasma (FRAP) as a measure of "antioxidant power": the FRAP assay. Anal Biochem 239:70-76. doi:10.1006/abio.1996.0292

Benzie IF, Szeto YT, Strain JJ, Tomlinson B (1999) Consumption of green tea causes rapid increase in plasma antioxidant power in humans. Nutr Cancer 34:83-87. doi:10.1207/\$15327914NC340112

Bers DM (2002) Cardiac excitation-contraction coupling. Nature 415:198-205. doi:10.1038/415198a

Bosch F, Sabater J, Valera A (1995) Insulin inhibits liver expression of the CCAAT/ enhancer-binding protein beta. Diabetes 44:267-271

Boudina S, Abel ED (2007) Diabetic cardiomyopathy revisited. Circulation 115: 3213-3223. doi:10.1161/CIRCULATIONAHA.106.679597

Cao G, Prior RL (1998) Comparison of different analytical methods for assessing total antioxidant capacity of human serum. Clin Chem 44:1309-1315

Chang S, Graham B, Yakubu F, Lin D, Peters JC, Hill JO (1990) Metabolic differences between obesity-prone and obesity-resistant rats. Am J Physiol 259:R1103-R1110

Chavez BEHRR (2006) Type 2 diabetes: insulin resistance, beta cell dysfunction, and other metabolic and hormonal abnormalities. In: Clinical Diabetes. Saunders Elsevier, PA, USA, pp 21-34

Cory S, Adams JM (2002) The Bcl2 family: regulators of the cellular life-or-death switch. Nat Rev Cancer 2:647-656. doi:10.1038/nrc883

Currie S, Smith GL (1999) Enhanced phosphorylation of phospholamban and downregulation of sarco/endoplasmic reticulum Ca2+ ATPase type 2 (SERCA 2) in cardiac sarcoplasmic reticulum from rabbits with heart failure. Cardiovasc Res 41:135-146

Davidoff AJ et al (2004) Sucrose-induced cardiomyocyte dysfunction is both preventable and reversible with clinically relevant treatments. Am J Physio Endocrinol Metab 286:E718-E724. doi:10.1152/ajpendo.00358.2003

de Pascual-Teresa S, Moreno DA, Garcia-Viguera C (2010) Flavanols and anthocyanins in cardiovascular health: a review of current evidence. Int J Mol Sci 11:1679-1703. doi:10.3390/ijms11041679

DeFronzo RA (2010) Insulin resistance, lipotoxicity, type 2 diabetes and atherosclerosis: the missing links. The Claude Bernard Lecture 2009. Diabetologia 53:1270-1287. doi:10.1007/s00125-010-1684-1

Diniz YS et al (2008) Conjugated linoleic acid and cardiac health: oxidative stress and energetic metabolism in standard and sucrose-rich diets. Eur J Pharmacol 579:318-325. doi:10.1016/j.ejphar.2007.11.008

Eckel RH (2005) Obesity. Circulation 111:e257-e259. doi:10.1161/01.CIR. 0000163653.38992.E5

Everard A, Cani PD (2013) Diabetes, obesity and gut microbiota best practice \& research. Clin Gastroenterol 27:73-83. doi:10.1016/j.bpg.2013.03.007

Fang CX, Dong F, Ren BH, Epstein PN, Ren J (2005) Metallothionein alleviates cardiac contractile dysfunction induced by insulin resistance: role of Akt phosphorylation, PTB1B, PPARgamma and c-Jun. Diabetologia 48:2412-2421. doi:10.1007/s00125-005-1940-y

Frank KF, Bolck B, Brixius K, Kranias EG, Schwinger RH (2002) Modulation of SERCA implications for the failing human heart. Basic Res Cardiol 97 Suppl 1:172-178

Galderisi M, Anderson KM, Wilson PW, Levy D (1991) Echocardiographic evidence for the existence of a distinct diabetic cardiomyopathy (the Framingham Heart study). Am J Cardiol 68:85-89

Hage M, Zantout MS, Azar ST (2011) Thyroid disorders and diabetes mellitus. J Thyroid Res 2011:439463. doi:10.4061/2011/439463

Hardie DG (2008) AMPK: a key regulator of energy balance in the single cell and the whole organism. Int J Obes 32 Suppl 4:S7-S12. doi:10.1038/ijo.2008.116

Hawley SA, Gadalla AE, Olsen GS, Hardie DG (2002) The antidiabetic drug metformin activates the AMP-activated protein kinase cascade via an adenine nucleotide-independent mechanism. Diabetes 51:2420-2425

International Diabetes Federation (2015) IDF Diabetes, 7 ed. International Diabetes Federation, Brussels, Belgium. http://www.diabetesatlas.org. Assessed 5 Oct 2015.

Kasuga M (2006) Insulin resistance and pancreatic beta cell failure. J Clin Invest 116:1756-1760. doi:10.1172/JCl29189

Koss KL, Grupp IL, Kranias EG (1997) The relative phospholamban and SERCA2 ratio: a critical determinant of myocardial contractility. Basic Res Cardiol 92 Suppl 1:17-24
Kranias EG, Bers DM (2007) Calcium and cardiomyopathies. Subcell Biochem 45:523-537

Laemmli UK (1970) Cleavage of structural proteins during the assembly of the head of bacteriophage T4. Nature 227:680-685

Lauterio TJ, Bond JP, Ulman EA (1994) Development and characterization of a purified diet to identify obesity-susceptible and resistant rat populations. J Nutr 124:2172-2178

Lebovitz HE (1999) Type 2 diabetes: an overview. Clin Chem 45:1339-1345

Lecomte $V$ et al (2010) A new role for sterol regulatory element binding protein 1 transcription factors in the regulation of muscle mass and muscle cell differentiation. Mol Cell Biol 30:1182-1198. doi:10.1128/MCB. 00690-09

Lee IC, Kim DY, Choi BY (2014) Antioxidative activity of blueberry leaf extract prevents high-fat diet-induced obesity in C57BL/6 mice. J Cancer Prev 19: 209-215. doi:10.15430/JCP.2014.19.3.209

Li L, Desantiago J, Chu G, Kranias EG, Bers DM (2000) Phosphorylation of phospholamban and troponin I in beta-adrenergic-induced acceleration of cardiac relaxation. Am J Physiol Heart Circ Physiol 278:H769-H779

Li H, Deng Z, Zhu H, Hu C, Liu R, Young JC, Tsao R (2012) Highly pigmented vegetables: anthocyanin compositions and their role in antioxidant activities. Food Res Int 46:250-259

Maisch B, Alter P, Pankuweit S (2011) Diabetic cardiomyopathy — fact or fiction? Herz 36:102-115. doi:10.1007/s00059-011-3429-4

Mazza G and Miniati E (1993) Anthocyanins in fruits, vegetables and grains. CRC Press Inc., Boca Raton

Mellor KM, Ritchie RH, Davidoff AJ, Delbridge LM (2010) Elevated dietary sugar and the heart: experimental models and myocardial remodeling. Can J Physiol Pharmacol 88:525-540. doi:10.1139/y10-005

Muniyappa R, Montagnani M, Koh KK, Quon MJ (2007) Cardiovascular actions of insulin. Endocr Rev 28:463-491. doi:10.1210/er.2007-0006

Perez de Heredia F, Garaulet M, Portillo MP, Zamora S (2006) Resistance to dietary obesity in rats given different high-energy diets. International journal for vitamin and nutrition research Internationale Zeitschrift fur Vitamin- und Ernahrungsforschung Journal international de vitaminologie et de nutrition 76:271-279. doi:10.1024/0300-9831.76.5.271

Prior RL et al (2003) Assays for hydrophilic and lipophilic antioxidant capacity (oxygen radical absorbance capacity (ORAC(FL))) of plasma and other biological and food samples. J Agric Food Chem 51:3273-3279. doi:10.1021/jf0262256

Ramirez-Correa GA, Murphy AM (2007) Is phospholamban or troponin I the "prima donna" in beta-adrenergic induced lusitropy? Circ Res 101:326-327. doi:10.1161/CIRCRESAHA.107.158873

Reaven GM (1988) Banting lecture 1988. Role of insulin resistance in human disease. Diabetes 37:1595-1607

Roberts CK, Barnard RJ, Sindhu RK, Jurczak M, Ehdaie A, Vaziri ND (2005) A high-fat, refined-carbohydrate diet induces endothelial dysfunction and oxidant/antioxidant imbalance and depresses NOS protein expression. J Appl Physiol 98:203-210. doi:10.1152/japplphysiol.00463.2004

Sewter C et al (2002) Human obesity and type 2 diabetes are associated with alterations in SREBP1 isoform expression that are reproduced ex vivo by tumor necrosis factor-alpha. Diabetes 51:1035-1041

Sharma V, McNeill JH (2006) Diabetic cardiomyopathy: where are we 40 years later? Can J Cardiol 22:305-308

Styskal J, Van Remmen H, Richardson A, Salmon AB (2012) Oxidative stress and diabetes: what can we learn about insulin resistance from antioxidant mutant mouse models? Free Radic Biol Med 52:46-58. doi:10.1016/j.freeradbiomed.2011.10.441

Takikawa M, Inoue S, Horio F, Tsuda T (2010) Dietary anthocyanin-rich bilberry extract ameliorates hyperglycemia and insulin sensitivity via activation of AMP-activated protein kinase in diabetic mice. J Nutr 140: 527-533. doi:10.3945/jn.109.118216

Towbin H, Staehelin T, Gordon J (1979) Electrophoretic transfer of proteins from polyacrylamide gels to nitrocellulose sheets: procedure and some applications. Proc Natl Acad Sci U S A 76:4350-4354

Tsuda T, Horio F, Uchida K, Aoki H, Osawa T (2003) Dietary cyanidin 3-O-beta-Dglucoside-rich purple corn color prevents obesity and ameliorates hyperglycemia in mice. J Nutr 133:2125-2130

Turnbaugh PJ, Backhed F, Fulton L, Gordon JI (2008) Diet-induced obesity is linked to marked but reversible alterations in the mouse distal gut microbiome. Cell Host Microbe 3:213-223. doi:10.1016/j.chom.2008.02.015 
van den Brom CE et al (2009) Altered myocardial substrate metabolism is associated with myocardial dysfunction in early diabetic cardiomyopathy in rats: studies using positron emission tomography. Cardiovasc Diabetol 8:39. doi:10.1186/1475-2840-8-39

Vasanji Z, Cantor EJ, Juric D, Moyen M, Netticadan T (2006) Alterations in cardiac contractile performance and sarcoplasmic reticulum function in sucrose-fed rats is associated with insulin resistance. Am J Physiol Cell Physiol 291:C772-C780. doi:10.1152/ajpcell.00086.2005

Wu T, Tang Q, Gao Z, Yu Z, Song H, Zheng X, Chen W (2013) Blueberry and mulberry juice prevent obesity development in C57BL/6 mice. PLoS One 8 , e77585. doi:10.1371/journal.pone.0077585

Ye G, Donthi RV, Metreveli NS, Epstein PN (2005) Overexpression of hexokinase protects hypoxic and diabetic cardiomyocytes by increasing ATP generation. Cardiovasc Toxicol 5:293-300

Zdychova J, Komers R (2005) Emerging role of Akt kinase/protein kinase B signaling in pathophysiology of diabetes and its complications. Physiol Res 54:1-16

Zhang R, Zhao J, Mandveno A, Potter JD (1995) Cardiac troponin I phosphorylation increases the rate of cardiac muscle relaxation. Circ Res 76:1028-1035

Zhang Z, Kou X, Fugal K, McLaughlin J (2004) Comparison of HPLC methods for determination of anthocyanins and anthocyanidins in bilberry extracts. J Agric Food Chem 52:688-691. doi:10.1021/jf034596w

\section{Submit your next manuscript to BioMed Central and we will help you at every step:}

- We accept pre-submission inquiries

- Our selector tool helps you to find the most relevant journal

- We provide round the clock customer support

- Convenient online submission

- Thorough peer review

- Inclusion in PubMed and all major indexing services

- Maximum visibility for your research

Submit your manuscript at www.biomedcentral.com/submit 